\title{
Rickettsial illnesses, a leading cause of acute febrile illness
}

\author{
Author: Ranjan Premaratna ${ }^{\mathrm{A}}$
}

Rickettsial illnesses, comprising mainly spotted fever group, typhus group and scrub typhus, are vector-borne re-emerging or newly emerging febrile illnesses where humans are an accidental dead-end host. They are a major cause of non-malarial febrile illnesses among returned travellers. They commonly present as an acute febrile illness and carry a characteristic entry wound (eschar) or a discrete erythematous maculo-popular rash based on the organism and the region. The illness severity is mainly dependent on the virulence of the rickettsial organism and delay in the diagnosis is known to cause severe illness with multi-organ involvement carrying high mortality. Almost all rickettsial infections respond to anti-rickettsial antibiotics such as doxycycline within 48-72 hours. Awareness of rickettsial illnesses and their various clinical presentations helps in early diagnosis and institution of appropriate treatment and hence prevent morbidity and mortality.

\section{Introduction}

Rickettsial illnesses, caused by obligate intracellular gram-negative coccobacili which multiply within eukaryotic cells, are re-emerging or newly emerging febrile illnesses where humans are accidental dead-end hosts. The genus of rickettsiae is divided into spotted fever group (SFG), typhus group and scrub typhus. All rickettsioses exist as zoonoses and are found in infected arthropods (ticks, mites, chiggers, fleas or lice), which generally serve as the natural hosts and can transmit the infection vertically to their next generation. These infections can be transmitted from arthropods to wild or domesticated animals such as dogs, cats and cattle.,

To date, SFG has nearly 15 pathogenic rickettsioses, including Rocky Mountain spotted fever (RMSF) caused by Rickettsia rickettsia, rickettsial pox caused by Rickettsia akari and Boutonneuse fever (also called as Kenya tick-bite fever, African tick typhus, Mediterranean spotted fever, Israeli spotted fever, Indian tick typhus and Marseilles fever). Typhus group includes Rickettsia prowazekii and Rickettsia typhi. Murine (endemic or flea-borne) typhus and scrub typhus are caused by Orientia tsutsugamushi and Orientia chuto. Orientia tsutsugamushi has three major serotypes: Karp, Gilliam and Kato. New or re-emerging rickettsioses described in the last few decades include tickborne lymphadenopathy (TIBOLA) and dermacentor-borne-necrosis-eschar-lymphadenopathy

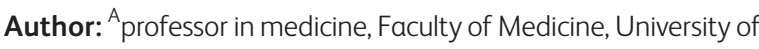
Kelaniya, Colombo, Sri Lanka, and honorary consultant physician, Colombo North Teaching Hospital, Ragama, Sri Lanka
(DEBONEL), related to Rickettsia slovaca infection, as well as lymphangitis-associated rickettsiosis attributed to Rickettsia sibricia infection. Furthermore, Ehrlichia organisms (the cause of human monocytic ehrlichiosis and Ehrlichia ewingii infection), Anaplasma phagocytophilum (the cause of human granulocytic anaplasmosis), and Bartonella species (the cause of Catscratch disease, relapsing fever, and Trench fever) are all related to the rickettsiae..$^{1-3}$

Although the clinical presentation of rickettsial infection is similar, the causative species and their illness presentation and severity

\section{Key points}

Rickettsial infections are re-emerging or emerging vectorborne diseases occurring in both tropical and temperate climates. In temperate climates incidence is rising during the summer months.

This is a neglected illness among returning travellers with acute febrile illness.

Early clinical features are like any other tropical febrile illness. Presence of a discrete erythematous rash that spares face or presence of an eschar helps in the diagnosis.

Basic haematology or biochemistry is not helpful in the diagnosis.

Delay in the diagnosis is known to result in severe illness: pneumonitis, myocarditis, encephalitis, acute renal failure and varying degrees of multi-organ involvement, sepsis and death.

Presence of acute hearing impairment (tinnitus or deafness) in a febrile patient predicts scrub typhus.

Clinical suspicion and early diagnosis and appropriate treatment is the key to prevent severe complications associated with rickettsial illnesses.

Empiric anti-rickettsial antibiotics are indicated when diagnostic facilities are not readily available, and clinical illness resolves within 48-f72 hours.

KEYWORDS: rickettsial infections, spotted fever, scrub typhus, fever with rash

DOI: 10.7861/clinmed.2021-0790 


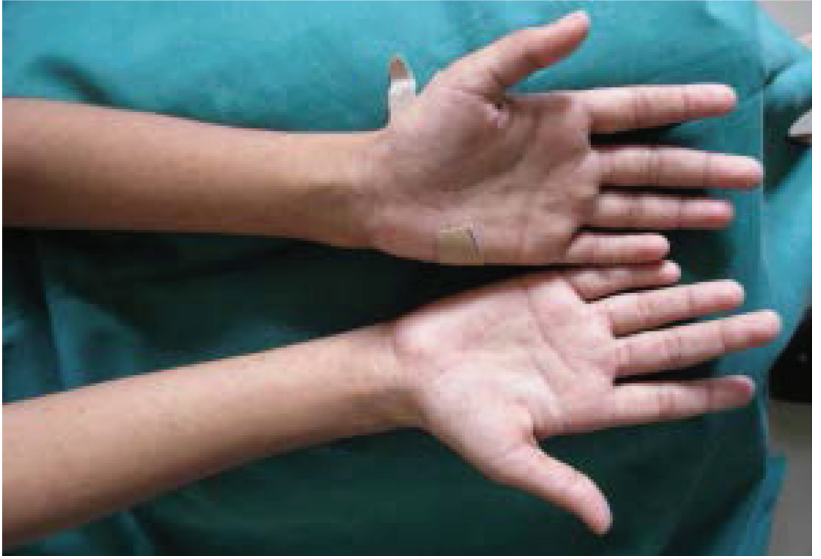

Fig 1. Discrete erythematous maculo-papular rash of spotted fever group rickettsioses

depends on the region. ${ }^{4}$ Therefore, it is important to recognise both the typical and atypical manifestations of rickettsial infection in each region to promptly diagnose and treat these infections, as they can be associated with significant morbidity and mortality. Rickettsial infections are a major cause of non-malarial febrile illnesses among returned travellers to endemic areas. ${ }^{3,5}$

\section{Presentation and diagnosis}

Rickettsial infection commonly accounts for an acute febrile illness with headache, body aches and joint pains similar to many other tropical illnesses. They have an incubation period of around 7-12 days. In SFG rickettsioses, towards the latter part of the first week, a discrete maculo-papular rash mainly involving the limbs, trunk, palms and soles, but sparing the face, is commonly seen (Fig 1).

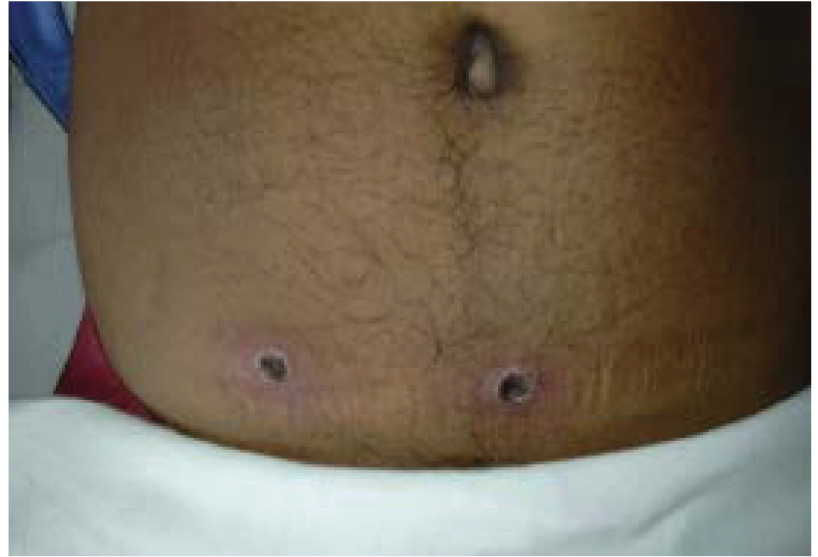

Fig 2. Eschars: the characteristic entry wound of scrub typhus and some spotted fever group rickettsioses.

A characteristic entry lesion or eschar (Fig 2) is commonly seen in scrub typhus and in some SFG rickettsioses such as African tick bite fever. They also can have a localised or generalised non-tender or mildly tender lymphadenopathy and varying degrees of hepatosplenomegaly. Although most patients recover even without treatment, delay in the diagnosis is known to result in serious multi-organ involvement (Fig 3), septicaemia and death. Features of severe illness include pneumonitis, acute respitory distress syndrome (ARDS), myocarditis, meningo-encephalitis and acute kidney injury. ${ }^{6,7}$ Acute sensorineural hearing impairments such as tinnitus or deafness have been described in association with scrub typhus, aiding the diagnosis of illness. ${ }^{8}$ In chronic undiagnosed illness, patients may develop manifestations suggestive of vasculitic phenomena such as transverse myelitis and cranial nerve palsies. Undiagnosed SFG caused by $R$ conorii indica in South

\section{Clinical features}

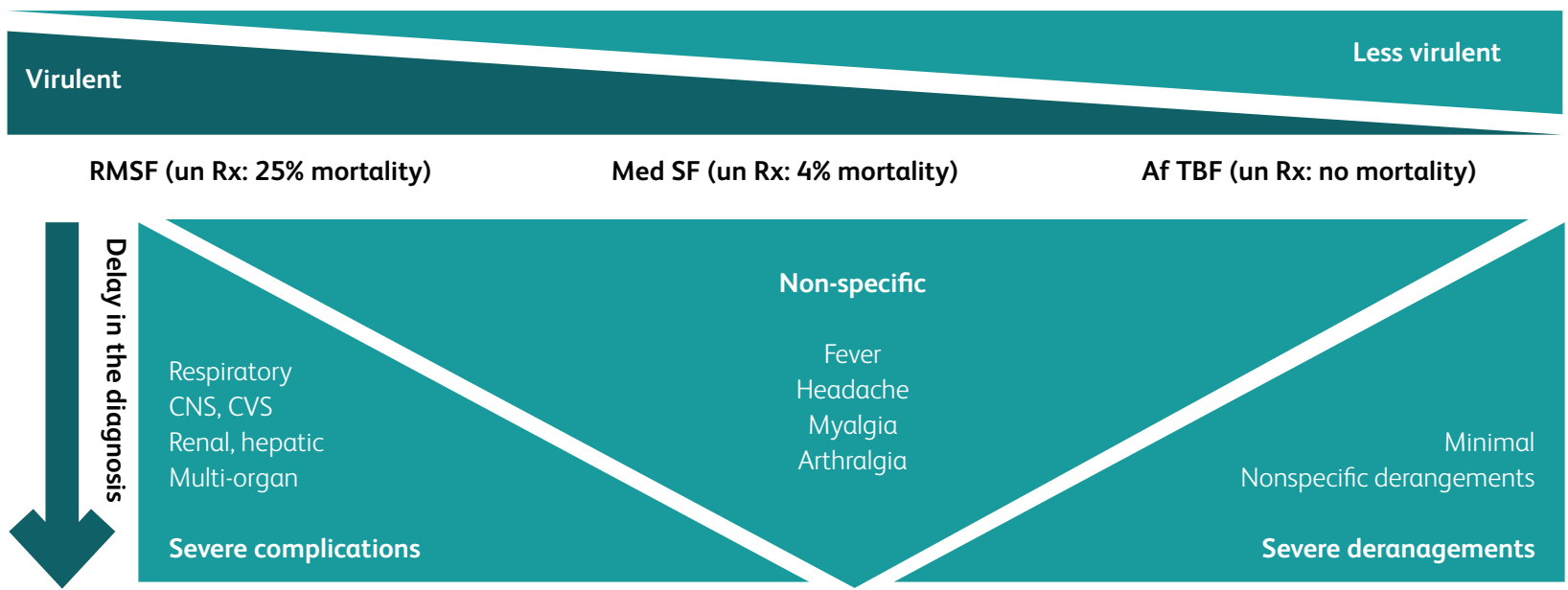

Fig 3. Summary of symptomatology of rickettsial illness. Af TBF, African tick bite fever; CNS, central nervous system; CVS, cardiovascular; Med SF, mediterranean spotted fever; RMSF, Rocky Mountain spotted fever. 


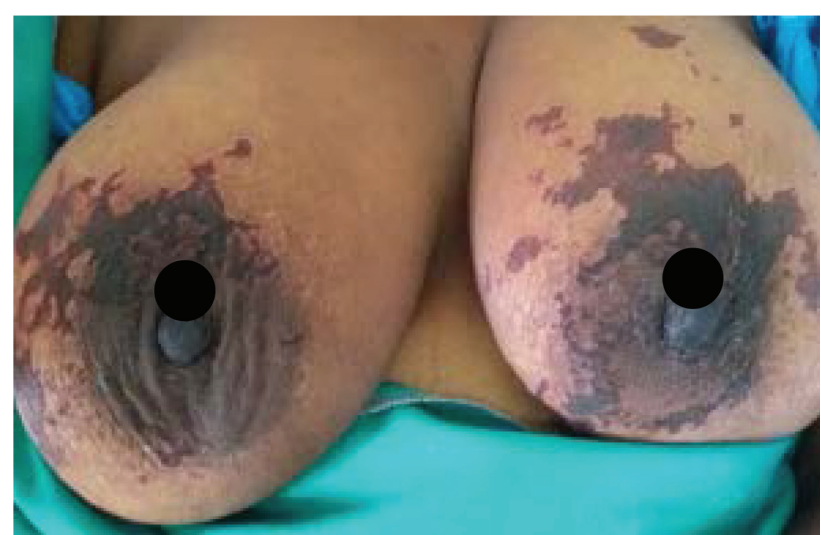

Fig 4. Purpura fulminans. ${ }^{9}$

Asian regions may result in purpura fulminans, characterised by rapidly progressive purpuric lesions developing into extensive areas of skin necrosis (Fig 4), and peripheral gangrene with laboratory evidence of consumptive coagulopathy; it is often fatal. ${ }^{9,10}$

\section{Diagnosis}

In endemic regions, rickettsial illness needs to be considered in any patient with an acute febrile illness, especially if they have a characteristic skin rash. However, careful and detailed clinical examination is needed in order to detect an eschar, which usually occur in moist areas of the body (such as axilla/groins or under breasts), in skin folds or along garment strap lines (Fig 5). ${ }^{11}$ Because of the association with ticks and other vectors, infections with rickettsiae are more common during warmer months and in people exposed to outdoor activities. In those who live in nonendemic areas, a recent visit to an endemic area, their activities during the visit such as eco-tourism, close contact with wild or domestic animals, sleeping or activities on grassy or scrub lands, and evidence of tick bites aid the diagnosis. In temperate climates, increasing incidence of rickettsial illness has been seen during warmer summer months. This has been attributed to migration of ticks from neighbouring endemic regions during summer months, their aggressiveness and close contact with humans during various outdoor activities. Therefore, rickettsial illnesses needs to be considered in the differential diagnosis of acute febrile illness even

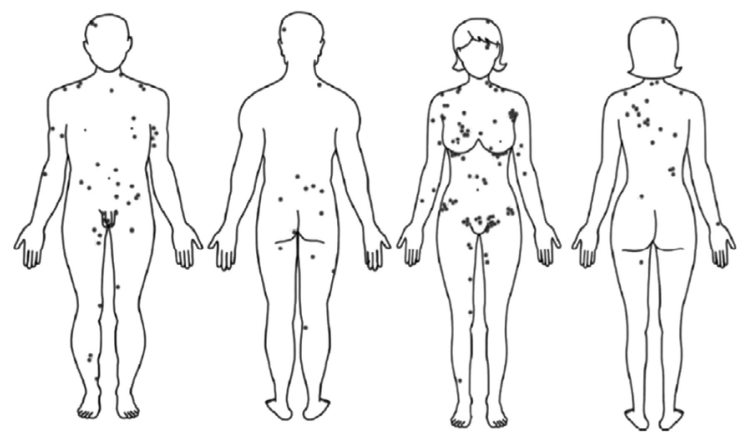

Fig 5. Distribution of eschars in the body. ${ }^{11}$ in temperate regions, especially during summer months. Careful documentation of unusual clinical presentations and attempts to identifying causative organisms and their treatment responsiveness are helpful in tackling these emerging or re-emerging rickettsial illnesses, because pathogenic to non-pathogenic rickettsial organisms are increasingly isolated in arthropod vector species in both endemic and non-endemic regions.

\section{Investigations}

Basic investigations usually fail to differentiate rickettsial infections from any other common acute febrile tropical illnesses; peripheral white blood cell count may be elevated, normal, or low and thus may not help to rule rickettsial infection in or out. ${ }^{12}$ Thrombocytopenia is a frequent finding. Hyponatremia, cerebrospinal fluid pleocytosis, a mild to moderate rise in inflammatory markers, or alterations in liver (AST/ALT) and renal (serum creatinine) biochemistry may be seen. However, severe derangements of above haematological or biochemical parameters are expected in patients with severe illness.

Confirmation of most rickettsial infections is based on serologic response, using immunofluorescence or ELISA-based assays to detect rising titres (especially in rickettsial disease endemic area) of IgG and IgM, in conjunction with a high index of clinical suspicion. ${ }^{13,14}$ However, there is a potential for negative serologic testing early in the course of illness. Therefore, early institution of anti-rickettsial antibiotics such as doxycycline is recommended on clinical suspicion alone given the high morbidity and mortality associated with rickettsial infections. ${ }^{15}$ A rapid clinical response to anti-rickettsial antibiotics such as doxycycline helps in the presumptive diagnosis when confirmatory diagnostics are not readily available. Culture of rickettsia using cell media in a laboratory with high level of biosafety (BSL III) facility and molecular diagnostic tests, such as PCR, on buffy coat samples or skin biopsies of eschars or vasculitic lesions help in the identification of causative emerging or re-emerging rickettsial organisms in a given geographic region.

\section{Treatment}

Most rickettsial diseases respond well to antibiotics such as tetracycline, doxycycline and azithromycin. Doxycycline $(100 \mathrm{mg}$, twice a day for 5-7 days) is recommended for treatment of all SFG infections. ${ }^{5} \mathrm{~A}$ similar treatment regime is adequate for most other rickettsial infections.

The $O$ tsutsugamushi Kato strain has been reported to be intrinsically resistant to quinolones. ${ }^{16}$ Reduced susceptibility to doxycycline and chloramphenicol has been reported northern Thailand. ${ }^{17}$ In such instances, rifampicin (300-450 mg, twice a day) for 1 week has been shown superior to doxycycline in terms of time to defervescence and relapse rates in scrub typhus. ${ }^{18}$ In areas where reduced susceptibility to tetracyclines is present, a single dose of azithromycin $(500 \mathrm{mg}$ ) may be equally effective as a seven-day course of doxycycline. ${ }^{19}$

Empiric treatment based on clinical suspicion alone is recommended for rickettsial infections that carry high mortality rates such as Rocky Mountain spotted fever, or when confirmatory facilities are not readily available. Chloramphenicol was historically used in the treatment of rickettsial infections; however, due to severe adverse side effects, its place is limited. Fluoroquinolones have been associated with worse outcomes and are not 
recommended for the treatment of rickettsial disease. Although doxycycline is not routinely utilised in children younger than 8 years of age, recent studies have failed to show harm during short courses. ${ }^{20}$ Azithromycin is recommended for treatment of rickettsial illness during pregnancy.

\section{Prognosis}

The severity of rickettsial infections varies based on the virulence of the rickettsial species and host immunity. While $R$ rickettsii and $R$ prowazekii are considered the most virulent species, $R$ africa is considered the mildest of all. Untreated Rocky Mountain spotted fever has mortality rates as high as $30 \%$. Therefore, timely diagnosis and treatment is the key to prevent morbidity and mortality associated with rickettsial illness. Starting appropriate antibiotics in the first week of illness is associated with very rapid recovery. In the absence of diagnostic facilities, empiric treatment with doxycycline helps to prevent morbidity and mortality. Delay in treatment may result in long-term sequelae such as hearing loss, bowel or bladder incontinence, variable paralysis of the lower extremities or gangrene of skin or extremities.

\section{Prevention and patient education}

Education on rickettsial illness and its mode of acquisition helps to reduce the incidence of illness. Prevention relies on avoidance of exposure to tick, lice, mite and flea bites, particularly when residing or traveling to endemic areas. Wearing long-sleeved shirts, trousers, socks and closed-toe shoes, use of insect repellents such as N,Ndiethyl-3-methylbenzamide (DEET) and permethrin-impregnated clothing and bedding, and inspection for insects after outdoor activities helps to minimise rickettsial infection. Currently, there is no effective vaccine for the most rickettsial infections including Rocky Mountain spotted fever. Once-a-week doses of doxycycline (200 $\mathrm{mg}$ ) can prevent scrub typhus, ${ }^{21,22}$ but there have been no studies on prophylaxis therapy for other rickettsioses.

\section{Conclusion}

Rickettsial infections are re-emerging or newly emerging infections that occur especially in the tropics but are also increasingly recognised in temperate climates due to global warming. They represent one of the common causes of non-malarial febrile illness among travellers, but are under-diagnosed. They usually present as an acute febrile illness and presence of a discrete erythematous maculo-papular rash and/or an eschar helps in the diagnosis. These infections show a very rapid clinical response to antibiotics such as doxycycline. Delay in the diagnosis and treatment results in multi-organ involvement and severe complications.

\section{References}

1 Walker DH. Rickettsiae. In: Baron S, editor, Medical Microbiology. 4th edition. Galveston (TX): University of Texas Medical Branch at Galveston; 1996.

2 Parola P, Paddock CD, Raoult D. Tick-borne rickettsioses around the world: emerging diseases challenging old concepts. Clin Microbiol $\operatorname{Rev} 2005 ; 18: 719-56$.
3 Ericsson CD, Jensenius M, Fournier P-E, Raoult D. Rickettsioses and the international traveler. Clin Infect Dis 2004;39:1493-19.

4 Walker DH. Rickettsiae and rickettsial infections: the current state of knowledge. Clin Infect Dis 2007:45:S39-44.

5 Aung AK, Spelman DW, Murray RJ, Graves S. Rickettsial infections in Southeast Asia: implications for local populace and febrile returned travelers. Am J Trop Med Hyg 2014;91:451-60.

6 Tsay RW, Chang FY. Serious complications in scrub typhus. J Microbiol Immunol Infect 1998;31:240-4.

7 Paris DH, Shelite TR, Day NP, Walker DH. Unresolved problems related to scrub typhus: a seriously neglected life-threatening disease. Am J Trop Med Hyg 2013;89:301-7.

8 Premaratna R, Chandrasena TGAN, Dassayake AS et al. Acute hearing loss due to scrub typhus: a forgotten complication of a reemerging disease. Clin Infect Dis 2006;42:e6-8.

9 Luke N, Munasinghe H, Balasooriya L, Premaratna R. Widespread subcutaneous necrosis in spotted fever group Rickettsioses from the coastal belt of Sri Lanka - a case report. BMC Infect Dis 2017; 17:278.

10 Katoch S, Kallappa R, Shamanur MB, Gandhi S. Purpura fulminans secondary to rickettsial infections: A case series. Indian Dermatol Online J 2016;7:24-8.

11 Kim D-M, Won KJ, Park CY et al. Distribution of eschars on the body of scrub typhus patients: a prospective study. Am J Trop Med Hyg 2007;76:806-809.

12 Bai PSP. Laboratory diagnosis of rickettsial infections. Pediatr Infect Dis 2015;7:85-7.

13 Graves S, Stenos ], Unsworth N, Nguyen C. Laboratory diagnosis of rickettsial infection. Aust J Med Sci 2006;27:39-44.

14 La Scola B, Raoult D. Laboratory diagnosis of rickettsioses: current approaches to diagnosis of old and new rickettsial diseases. J Clin Microbiol 1997;35:2715-27.

15 Raoult D, Drancourt M. Antimicrobial therapy of rickettsial diseases. Antimicrob Agents Chemother 1991;35:2457-62.

16 Tantibhedhyangkul W, Angelakis E, Tongyoo N et al. Intrinsic fluoroquinolone resistance in Orientia tsutsugamushi. Int J Antimicrob Agents 2010;35:338-41.

17 Watt G, Chouriyagune C, Ruangweerayud R et al. Scrub typhus infections poorly responsive to antibiotics in northern Thailand. Lancet 1996;348:86-9.

18 Watt G, Kantipong P, Jongsakul K et al. Doxycycline and rifampicin for mild scrub-typhus infections in northern Thailand: a randomised trial. Lancet 2000;356:1057-61.

19 Kim Y-S, Yun H-J, Shim SK et al. A comparative trial of a single dose of azithromycin versus doxycycline for the treatment of mild scrub typhus. Clin Infect Dis 2004:39:1329-35.

20 Purvis J], Edwards MS. Doxycycline use for rickettsial disease in pediatric patients. Pediatr Infect Dis ] 2000;19:871-4.

21 Olson JG, Bourgeois AL, Fang RC, Coolbaugh JC, Dennis DT. Prevention of scrub typhus. Prophylactic administration of doxycycline in a randomized double blind trial. Am J Trop Med Hyg 1980;29:989-97.

22 Twartz JC, Shirai A, Selvaraju G et al. Doxycycline prophylaxis for human scrub typhus. J Infect Dis 1982;146:811-8.

Address for correspondence: Prof R Premaratna,

Department of Medicine, Faculty of Medicine, PO Box 6, Thalagolla Road, Ragama, Sri Lanka.

Email: ranjanp64@kln.ac.Ik 